\title{
Lumbar Disc Degenerative Disorder
}

National Cancer Institute

\section{Source}

National Cancer Institute. Lumbar Disc Degenerative Disorder. NCI Thesaurus. Code C27154.

Any degenerative disorder affecting one or more vertebral discs of the lumbar spine. 